\title{
EDITORIAL
}

\section{LA PREVENCIÓN CARDIOVASCULAR EN ESPAÑA. PROMOVIENDO EL USO DE LAS RECOMENDACIONES}

\section{Fernando Villar Álvarez $(1,2)$}

(1) Escuela Nacional de Sanidad. Instituto de Salud Carlos III. Ministerio de Sanidad y Consumo.

(2) Departamento de Medicina Preventiva y Salud Pública. Facultad de Medicina. Universidad Autónoma de Madrid.

\section{SITUACIÓN CARDIOVASCULAR EN EL MUNDO}

Las enfermedades cardiovasculares constituyen un problema de salud de primer orden en todo el mundo, ya que a su importancia capital en los países desarrollados se une su creciente relevancia en los países en vías de desarrollo, en los que tienen una «doble carga» al sumarse a la persistente amenaza de las enfermedades transmisibles la aparición de las enfermedades no transmisibles. En todo el mundo 16,7 millones de muertes se deben a las enfermedades cardiovasculares ${ }^{1}$. Además, cinco de las diez principales amenazas mundiales para la salud están relacionadas con las enfermedades no transmisibles, como la hipertensión arterial, el tabaquismo, el consumo de alcohol, la hipercolesterolemia y la obesidad o el sobrepeso.

En Europa las enfermedades cardiovasculares son la principal causa de muerte ${ }^{2}$, tienen una alta incidencia ${ }^{3}$, son una fuente muy importante de discapacidad y tienen una gran repercusión en los costes de la asistencia sanitaria. Además, los principales factores de riesgo cardiovascular son muy prevalentes en los países europeos ${ }^{4}$.

\section{SITUACIÓN CARDIOVASCULAR EN ESPAÑA}

Del mismo modo, en España, la relevancia de las enfermedades cardiovasculares viene dada por su elevada mortalidad (produce el $35 \%$ de todas las defunciones, causando cerca de 125.000 cada año) y morbilidad (más de 560.000 enfermos dados de alta y de cinco millones de estancias hospitalarias al año), la alta prevalencia de sus principales factores de riesgo en la población española y su elevada repercusión socioeconómica (la atención a las enfermedades cardiovasculares es responsable del $15 \%$ de los costes sanitarios totales) $)^{5}$.

En España, aunque comparativamente con otros países las enfermedades cardiovasculares tengan una morbilidad y mortalidad más bajas, son la primera causa de muerte. El riesgo de morir por las enfermedades del aparato circulatorio está disminuyendo en España desde mediados de los años setenta, sobre todo debido al descenso de la mortalidad cerebrovascular. Sin embargo, a causa fundamentalmente del envejecimiento de la población, el número de muertes por coronariopatía ha aumentado. Por ello, el impacto demográfico, sanitario y social de estas enfermedades aumentará a lo largo de las próximas décadas.

En España hay importantes diferencias geográficas en la mortalidad cardiovascular, presentándose los valores más altos en Canarias y en las regiones peninsulares del sur y levante ${ }^{6}$. Asumiendo que una parte importante de las mismas se deben a factores 
ambientales, estas diferencias geográficas sugieren un importante potencial de prevención de las enfermedades cardiovasculares, que incluso puede alcanzar el $50 \%$.

En nuestro país la prevalencia de los principales factores de riesgo cardiovascular es elevada. El 34\% de los españoles mayores de 16 años fuma. Además, el tabaquismo muestra una tendencia desfavorable en mujeres jóvenes. No hay que olvidar que el tabaco produce aproximadamente 52.000 muertes al año en España (16\% de todas las defunciones $)^{7}$ y que es la primera causa de enfermedad, discapacidad y muerte prematuras y evitables en la población española. Al igual que otros países desarrollados, España está experimentando una epidemia de obesidad, tanto en adultos como en niños. En la actualidad aproximadamente 28.000 muertes cada año ( $8,5 \%$ de todas las defunciones) son atribuibles a la obesidad. Aproximadamente la mitad de la población española presenta valores de colesterol en sangre elevados (más de $200 \mathrm{mg} / \mathrm{dl}$ ), y la mayoría de las personas desconoce este hecho, e incluso la mayor parte de las personas que tienen alto riesgo cardiovascular no recibe tratamiento hipolipemiante. El porcentaje de población con hipertensión arterial es aproximadamente el $40 \%$, el cual aumenta con la edad y alcanza el $68 \%$ en las personas mayores de 60 años. En España el porcentaje de adultos que dedican más de 5 horas a actividades físicas cada semana es, tras Portugal, el más bajo de la Unión Europea. Estos datos son de extraordinaria importancia porque la actividad física regular es probablemente, junto a la abstinencia del tabaco, la medida más beneficiosa para la promoción de la salud, en particular para el control de la epidemia de obesidad, que además conduce a la elevación de otros factores de riesgo cardiovascular, como la dislipemia, la hipertensión arterial y la diabetes. En España el porcentaje de población con diabetes se estima en un $6 \%$ para los grupos de edad 30-65 años, y del 10\% para los grupos de 30-89 años. También la frecuencia de diabetes está aumentando y una parte muy importante de las personas diabéticas desconoce que lo son. Igualmente, la dieta de los españoles ha experimentado cambios asociados al desarrollo económico, aunque todavía se ajusta, en general, al patrón de dieta mediterránea considerada saludable ${ }^{5}$.

Además, el grado de control de la hipertensión arterial y de las dislipemias, aunque ha mejorado en los últimos años, es todavía bajo. En la actualidad sólo el $16 \%$ de la población hipertensa española tienen controlada su presión arterial de forma óptima. En las personas con enfermedad cardiovascular previa el control de los factores de riesgo cardiovascular es deficiente y claramente mejorable, tanto en el ámbito hospitalario como en el de la atención primaria de salud. Además el progreso realizado en los últimos años en el control de dichos factores en pacientes hospitalizados ha sido escaso o incluso ha empeorado para algunos factores, excepto para la dislipemia, que ha mejorado sustancialmente.

\section{ETIOPATOGENIA Y FACTORES DE RIESGO}

La aterosclerosis subyace a las principales causas de morbilidad y mortalidad cardiovascular. Esta se desarrolla lentamente desde las primeras décadas de la vida y es generalmente avanzada y difícilmente reversible cuando aparecen los síntomas. Por ello, el tratamiento en etapas avanzadas es fundamentalmente paliativo más que curativo. A esto se añade el hecho de que aproximadamente dos de cada tres muertes por infarto agudo de miocardio ocurren antes de llegar al hospital ${ }^{8}$, lo que refuerza el papel de la prevención primaria y secundaria para disminuir la mortalidad por cardiopatía isquémica, ya que la disminución de la letalidad por mejora del cuidado hospitalario va a tener un impacto limitado.

El desarrollo de la enfermedad coronaria está íntimamente relacionado con los estilos 
de vida y los factores de riesgo asociados y está claramente establecido que su modificación y control pueden retrasar la aparición de la enfermedad coronaria antes y después de que se hayan producido eventos clínicos.

\section{ESTRATEGIAS DE SALUD}

Acorde con su importancia, las enfermedades cardiovasculares son objetivo prioritario de las estrategias de salud desarrolladas por la Organización Mundial de la Salud. En la Estrategia de Salud para Todos en el año 2000 se formulaba como objetivo número ${ }^{9}$, reducir las enfermedades cardiovasculares, señalando que para dicho año la mortalidad por enfermedades del aparato circulatorio debería haberse reducido, en el caso de las personas mayores de 65 años, por lo menos en un $15 \%$ y debería haberse progresado en la mejora de la calidad de vida de todas las personas que sufran enfermedades cardiovasculares9. Posteriormente, en el objetivo número 8 de Salud para Todos en el siglo XXI, se menciona que la mortalidad por enfermedades cardiovasculares en las personas mayores de 65 años se deberá reducir para el año 2020 como promedio al menos un $40 \%$, en especial en los países que tienen una mortalidad muy elevada ${ }^{10}$.

\section{ESTRATEGIAS Y RECOMENDACIONES INTERNACIONALES DE SALUD CARDIOVASCULAR}

Cabe resaltar las Conferencias Internacionales de Salud Cardiovascular, cuya primera reunión en 1992 dio lugar a la Declaración de Victoria, que intenta representar a diversos países y a muchas disciplinas científicas con una Llamada a la Acción y un Marco de Política para todos los países del mundo. Esta declaración se basa en la convicción de que se dispone de los medios necesarios para prevenir o reducir sustancialmente la carga de las enfermedades cardiovasculares ${ }^{11}$.
Se han publicado numerosos documentos e informes de recomendaciones para la prevención de las enfermedades cardiovasculares y para el control de sus principales factores de riesgo, elaborados por organismos internacionales o por instituciones o sociedades científicas internacionales y nacionales (Estados Unidos, distintos países europeos, Australia y Nueva Zelanda, entre otros).

La Organización Mundial de la Salud publicó en 1982 el primer informe sobre Prevención de la cardiopatía coronaria ${ }^{12}$, con el objetivo de impulsar el desarrollo de programas de prevención y lucha contra las enfermedades cardiovasculares. Éstos debían tener tres componentes: una estrategia poblacional, una estrategia de riesgo elevado y la prevención secundaria. Posteriormente, en 1986, publicó un informe sobre Prevención y lucha contra las enfermedades cardiovasculares en la comunidad ${ }^{13}$ que incluye estrategias de la lucha contra la hipertensión arterial, los accidentes cardiacos, la fiebre reumática y la cardiopatía reumática desde una perspectiva comunitaria.

Consciente de la importancia de la dieta en la aparición de las enfermedades no transmisibles, la Organización Mundial de la Salud publicó en 1990 un informe sobre Dieta, nutrición y prevención de enfermedades crónicas $^{14}$, actualizado en $2003^{15}$. En él se propugna la necesidad de formular políticas alimentarias y nutricionales nacionales para prevenir o minimizar importantes problemas de salud. En la 57 ${ }^{\mathrm{a}}$ Asamblea Mundial de la Salud, celebrada en 2004, se ha aprobado una Estrategia Mundial sobre Régimen Alimentario y Actividad Física, que pretende promover y proteger la salud orientando la creación de un entorno favorable para la adopción de medidas sostenibles en el ámbito individual, comunitario, nacional y mundial que, en conjunto, den lugar a una reducción de la morbilidad y la mortalidad asociadas a una alimentación poco sana y a la falta de actividad física. 
La propia Organización Mundial de la Salud se ocupa de la hipertensión arterial desde hace décadas, con recomendaciones específicas sobre un factor de riesgo. Ya en 1958 el Comité de Expertos en Enfermedades Cardiovasculares e Hipertensión analizó su clasificación y criterios diagnósticos ${ }^{16}$. Tres años más tarde se describieron las etapas de la hipertensión y se propusieron recomendaciones terapéuticas para frenar la evolución de la enfermedad (prevención secundaria $)^{17}$. En 1978, un tercer informe se ocupó de la epidemiología, la prevención y el control de la hipertensión ${ }^{18}$. En 1994 el Comité de Expertos en Control de la Hipertensión examinó la epidemiología y las estrategias para la prevención y el tratamiento, con el objetivo de incorporar el control de la hipertensión a los programas para reducir el riesgo cardiovascular total, resaltar la estrategia poblacional en la prevención primaria de la hipertensión y proponer opciones terapéuticas. También se hizo hincapié en la importancia de la presión arterial sistólica y en las recomendaciones para personas mayores, y se incorporaron consideraciones sobre la eficacia y el coste de la prevención y el tratamiento a la hora de elegir las estrategias óptimas ${ }^{19}$.

Más recientemente, en los años 1999 y 2003, la Organización Mundial de la Salud junto con la Sociedad Internacional de Hipertensión (WHO-ISH), elaboró recomendaciones para el manejo de la hipertensión arterial, que establecen pautas de actuación para su detección, tratamiento, seguimiento y control. Dichas recomendaciones proponen estimar el riesgo cardiovascular para indicar el tratamiento de la hipertensión arterial e insisten en que su abordaje terapéutico debe realizarse sin olvidar el tratamiento concomitante de otros factores de riesgo cardiovascular $^{20,21}$.

\section{RECOMENDACIONES CARDIOVASCULARES EN EEUU}

En Estados Unidos, auspiciado por el Instituto Nacional de Salud Norteamericano
(NIH), en 1988 se publicó el primer informe del Comité de Expertos sobre Detección, Evaluación y Tratamiento de la Hipercolesterolemia en Adultos (ATP I) ${ }^{22}$, que propuso un enfoque clínico sistemático para el tratamiento de las concentraciones séricas elevadas de colesterol en adultos. Entre sus principales características cabe destacar que identifica a las lipoproteínas de baja densidad (LDL) como principal objetivo del tratamiento hipolipemiante, señala que la terapéutica dietética constituye la primera línea de tratamiento de la hipercolesterolemia, reservando el tratamiento farmacológico para las personas de alto riesgo, y subraya que se debe individualizar el tratamiento de cada persona. Posteriormente, en 1990, se publicó el informe del Comité de Estandarización de Laboratorio, en el que se hicieron recomendaciones para mejorar la exactitud de las determinaciones del colesterol, más tarde el informe del Comité de Población, que propuso un enfoque de salud pública, y en 1991 el informe del Comité Pediátrico ${ }^{23}$. Estos cuatro informes conforman la estrategia del National Cholesterol Education Program (NCEP) para el control de la hipercolesterolemia en Estados Unidos. En 1993 se publicó el Segundo Informe Comité de Tratamiento de Adultos (ATP II) ${ }^{24}$, que actualizó las recomendaciones e introdujo algunas novedades, entre las que se puede señalar que prioriza la actuación en los pacientes que ya han padecido una enfermedad aterosclérótica, presta más atención a las lipoproteínas de alta densidad (HDL) y hace mayor énfasis en la reducción del peso y en la actividad física como elementos del tratamiento. En 2001 se publicó la última actualización del Panel de Tratamiento de Adultos III (ATP III) ${ }^{25}$. Entre las características nuevas del ATP III cabe reseñar su focalización en casos con múltiples factores de riesgo: utiliza las proyecciones de Framingham de riesgo de infarto a 10 años para identificar a determinados pacientes con múltiples factores de riesgo para tratamiento intensivo, considera la diabetes como equivalente de riesgo de cardiopatía isquémica, y hace énfasis 
en las personas con síndrome metabólico en las que los cambios terapéuticos deben ser más intensos en el estilo de vida. También modifica la clasificación de lípidos y lipoproteínas, fija nuevas recomendaciones para el cribado y detección, intensifica la intervención en estilos de vida y presenta nuevas estrategias para promover el cumplimiento terapéutico.

Centrados en la hipertensión arterial se dispone de los informes del Joint National Committee (JNC). En 1997, el VI Informe del JNC hizo énfasis en la estratificación del riesgo cardiovascular y señaló la oportunidad de conseguir niveles de control de la presión arterial más estrictos $(<130 / 85 \mathrm{~mm} \mathrm{Hg})$ en presencia de diabetes, insuficiencia renal o insuficiencia cardiaca ${ }^{26}$. En 2003 se publicó el séptimo informe ${ }^{27}$. En él se introduce el concepto de «prehipertensión» (presión arterial, 120-139/80-89 mm Hg) para sensibilizar a la población general y a los profesionales sanitarios para que apliquen, lo antes posible, estrategias efectivas dirigidas a una vida más sana a través de la prevención de la hipertensión y de la enfermedad cardiovascular relacionada con ésta. Además, elimina la hipertensión de fase 3 , uniéndola con la de fase 2, a diferencia de las recomendaciones europeas sobre hipertensión, que sí distinguen entre $\operatorname{ambas}^{28}$. En el JNC 7, también se señala que la presión arterial sistólica es más importante que la diastólica como factor de riesgo cardiovascular, excepto, quizá, en las personas más jóvenes.

También son de gran interés las recomendaciones publicadas por la Asociación Norteamericana del Corazón sobre Salud cardiovascular en la infancia ${ }^{29}$, las Directrices para la prevención primaria de la enfermedad cerebrovascular ${ }^{30}$, y el recientemente publicado documento, elaborado conjuntamente con las Sociedades Norteamericanas del Cáncer y de Diabetes, sobre la Prevención del cáncer, las enfermedades cardiovasculares y la diabetes ${ }^{31}$. Hay que resaltar el hecho de que en un mismo documento se contemplen estas tres enfermedades. Las enfermedades cardiovasculares, el cáncer y la diabetes son responsables aproximadamente de dos de cada tres muertes que se producen en Estados Unidos, y comparten algunos de sus principales factores de riesgo: tabaquismo, dieta inadecuada e inactividad física, por lo cual se puede hacer una sinergia de esfuerzos para su prevención.

\section{RECOMENDACIONES CARDIOVASCULARES EN EUROPA}

En el ámbito europeo se han publicado numerosos documentos de recomendaciones para la prevención de las enfermedades cardiovasculares y para el control de sus principales factores de riesgo, elaborados por instituciones o sociedades científicas europeas y nacionales.

En Europa los antecedentes de recomendaciones cardiovasculares los encontramos cuando la Sociedad Europea de Arteriosclerosis publicó un documento de estrategias para la prevención de la cardiopatía isquémica en $1987^{32}$, el cual está actualizado posteriormente en $1992^{33}$.

Las actuales Recomendaciones Europeas, cuya versión resumida se publican traducidas al español en este número de la Revista Española de Salud Pública ${ }^{34}$, tienen su origen en 1994, cuando se publicaron por primera vez como esfuerzo conjunto de tres sociedades científicas: la Sociedad Europea de Cardiología, la Sociedad Europea de Aterosclerosis y la Sociedad Europea de Hipertensión ${ }^{35}$. Estas primeras recomendaciones se centraron en la prevención de la cardiopatía isquémica en la práctica clínica. Es importante señalar que estas recomendaciones en lugar de centrar su atención en un factor de riesgo como la hipercolesterolemia o la hipertensión arterial, como habían hecho los NCEP o los JNC respectivamente, se dirigen a una enfermedad, la cardiopatía isquémica, que es la principal causa de 
muerte en los países del norte y centro de Europa. Cabe destacar otros dos aspectos relevantes de estas primeras recomendaciones. Primero, que establecen unas prioridades de la prevención de la enfermedad coronaria en la práctica clínica. Segundo, que se hace énfasis en la valoración del riesgo total que permita una aproximación más real al individuo. Este enfoque tiene en cuenta tres hechos importantes: que la etiología de la enfermedad coronaria es multifactorial, que los factores de riesgo tienen efectos multiplicativos y que se trata a personas y no a factores de riesgo aislados. Para estimar el riesgo absoluto de padecer un evento coronario a los 10 años se emplea el riesgo coronario, calculado en una función del riesgo derivada del Estudio de Framingham.

En 1998 se publicaron las Segundas Recomendaciones Europeas ${ }^{36}$, sumándose a su elaboración la Sociedad Europea de Medicina General/Medicina de Familia, la Red Europea del Corazón y la Sociedad Internacional de Medicina Conductual. Estas recomendaciones establecieron los objetivos para los estilos de vida, los factores de riesgo y el tratamiento para la prevención de la coronariopatía, fijaron las prioridades para la prevención de la enfermedad coronaria en la práctica clínica, utilizando las conclusiones el Estudio de Framingham para la valoración del riesgo coronario total.

Las Terceras Recomendaciones Europeas de Prevención Cardiovascular se publicaron en $2003^{37}$. A su redacción se añadieron la Asociación Europea para el estudio de la Diabetes y la Federación Internacional de Diabetes Europa. El objetivo de este documento es resumir desde una perspectiva clínica los principales aspectos sobre la prevención de la enfermedad cardiovascular para facilitar su implantación. Los pacientes con enfermedad cardiovascular conocida y los de alto riesgo serán los más beneficiados, ya que la acción preventiva es máxima en estos grupos. Al mismo tiempo, hay que tener en cuenta que la mayoría de falleci- mientos por enfermedad cardiovascular se producirán en personas con riesgo moderado o bajo porque son más numerosos que aquellos con alto riesgo. Por tanto, la estrategia de alto riesgo propuesta en este documento debe complementar y reforzar las acciones preventivas poblacionales dirigidas al conjunto de toda la población.

Hay una novedad clave que introducen estas recomendaciones respecto a las anteriores. Este cambio se aprecia ya en el título. Pasa de la prevención de la cardiopatía isquémica a la prevención cardiovascular. Esto hace que en lugar de utilizar el riesgo coronario derivado del estudio de Framingham, se emplee el riesgo de muerte cardiovascular obtenido del proyecto Systematic Coronary Risk Evaluation (SCORE) ${ }^{38}$. Así se estima el riesgo de muerte cardiovascular por enfermedad isquémica del corazón, enfermedad cerebrovascular, arteriopatía periférica y otras enfermedades vasculares ateroscleróticas a partir de distintos estudios de cohortes de doce países europeos, incluyendo tres cohortes españolas. Existen diferentes tablas para países con riesgo elevado y para los que tienen riesgo bajo, entre los que se encuentra España. Además, existe la posibilidad de utilizar tablas con o sin cHDL. Consecuentemente, al cambiar las tablas se cambia el criterio para determinar el riesgo elevado en personas que no han padecido un episodio cardiovascular previo. Mientras en las primeras y segundas recomendaciones se definía como de riesgo alto a las personas que presentaban un riesgo coronario total igual o superior al $20 \%$ en los próximos diez años (riesgo de padecer angina de pecho estable, infarto de miocardio silente o clínicamente manifiesto, insuficiencia coronaria - angina inestable- y muerte por enfermedad coronaria). Ahora, utilizando las tablas del SCORE, se considera pacientes de riesgo elevado a aquellos que tienen una probabilidad igual o superior al $5 \%$ de morir por cardiopatía isquémica, enfermedad cerebrovascular y arteriopatía periférica en los próximos diez años. El 
escaso tiempo transcurrido desde la publicación de estas nuevas recomendaciones hace que todavía no exista una experiencia suficiente en el uso de las tablas del SCORE para conocer las consecuencias prácticas de su aplicación clínica.

Como recomendaciones europeas específicamente sobre un factor de riesgo, en 2003, la Sociedad Europea de Hipertensión y la Sociedad Europea de Cardiología publicaron sus propias guías sobre hipertensión $\operatorname{arterial}^{28}$. De esta forma daban respuesta a la sugerencia de las directrices de la WHO-ISH de 1999 relativas a la elaboración, por parte de los expertos regionales, de recomendaciones específicamente dirigidas al abordaje del problema en las personas de su propia región. Por ello, estas recomendaciones fueron aprobadas por la Sociedad Internacional de Hipertensión. Muy poco tiempo después, como se ha indicado, aparecen las nuevas guías 2003 de la Organización Mundial de la Salud junto con la Sociedad Internacional de Hipertensión $^{21}$.

De ámbito nacional se pueden señalar las guías británicas. En 1998 cuatro sociedades científicas británicas publicaron un documento conjunto sobre la prevención de la cardiopatía isquémica en la práctica clíni$\mathrm{ca}^{39}$. Específicamente sobre hipertensión arterial, la Sociedad Británica de Hipertensión publicó sus directrices en $1999^{40}$. A destacar las magníficamente documentadas directrices clínicas nacionales escocesas del Scottish Intercollegiate Guidelines Network (SIGN), elaboradas según el nivel de evidencia sobre Lípidos y prevención primaria de la cardiopatía isquémica de $1999^{41}$ y de Prevención secundaria de la cardiopatía isquémica de $2000^{42}$.

\section{RECOMENDACIONES CARDIOVASCULARES EN ESPAÑA}

Dada la necesidad de actuar de manera efectiva para paliar el efecto de las enferme- dades cardiovasculares en España, inicialmente en pos de la consecución del objetivo número 9 de la Estrategia de Salud para Todos en el año 2000, de la Organización Mundial de la Salud, relativo a la disminución de la mortalidad debida a las enfermedades cardiovasculares, desde el Ministerio de Sanidad y Consumo se ha potenciado la elaboración de documentos para consensuar prácticas racionales y homogéneas de actuación ante algunos de los principales factores de riesgo de las enfermedades cardiovasculares. Para ello se contaba con las experiencias previas realizadas por organismos internacionales y por otros países, ya mencionadas, y por sociedades científicas españolas. En 1989 se publicó el Consenso para el Control de la Colesterolemia en España ${ }^{43}$, gracias a la colaboración del Ministerio de Sanidad y Consumo con la Sociedad Española de Cardiología y catorce entidades científicas más. Una década más tarde se publicó el documento de Control de la Colesterolemia en España en el año $2000^{44}$, con la Sociedad Española de Cardiología, la Sociedad Española de Arteriosclerosis y veintiuna sociedades científicas e instituciones involucradas en el tema. Su objetivo era llevar el conocimiento científico existente a la práctica clínica, adaptando las recomendaciones a los condicionantes diferenciales de nuestra realidad socio-sanitaria. Tal como indica el subtítulo del documento, pretende servir de instrumento para la prevención cardiovascular a través del control de la colesterolemia. Por ello, incorpora en prevención primaria el concepto de riesgo coronario, empleando para su cálculo las tablas de riesgo del estudio de Framingham propuestas por las Segundas Recomendaciones Europeas ${ }^{36}$.

Referente a la hipertensión, en 1990 se publicó el Consenso para el control de la Hipertensión Arterial ${ }^{45}$, fruto de la colaboración del Ministerio de Sanidad y Consumo con la Liga Española para la Lucha contra la Hipertensión Arterial, la Sociedad Española de Cardiología y dieciséis sociedades e instituciones de este ámbito. Se formularon 
directrices para la detección, tratamiento y seguimiento, así como la prevención de la hipertensión arterial y sus complicaciones. El último Documento de Control de la Hipertensión Arterial en España se publicó en 1996, con la participación de la Sociedad Española de Hipertensión-Liga Española para la Lucha contra la Hipertensión Arterial y veintisiete sociedades científicas e instituciones $^{46}$.

Además de la labor de redactar estos documentos de control de la colesterolemia y la hipertensión arterial, se hizo un gran esfuerzo para difundir las recomendaciones, con el objetivo de mejorar el grado de conocimiento, las actitudes y finalmente las conductas de la práctica clínica. La estrategia de introducción y difusión de las recomendaciones incluyó la edición de más de 70.000 ejemplares, y su publicación tanto en revistas científicas biomédicas de elevado impacto como en revistas de divulgación. También se presentó en Congresos de Sociedades Científicas y se hicieron más de cien seminarios específicos de presentación y discusión de las recomendaciones en toda España, en los que se empleó el material didáctico audiovisual elaborado al efecto. En estos seminarios se evaluaron los cambios en los conocimientos y actitudes sobre estos problemas ${ }^{47}$. Globalmente, el impacto de los consensos para el control de la colesterolemia y la hipertensión arterial en España fue razonable $(60 \%)$, en términos de grado de conocimiento, aunque el impacto en la práctica clínica fue menor ${ }^{48}$.

Como una estrategia de desarrollo de las recomendaciones formuladas en los documentos para el control de la colesterolemia y de la hipertensión arterial en España, se publicaron las «Directrices para la elaboración de programas de prevención primaria de enfermedades cardiovasculares ${ }^{49}$, las cuales pretendían proporcionar criterios para la elaboración y el impulso de los programas de prevención primaria de las enfermedades cardiovasculares, a través de la detección y control individual de sus principales factores de riesgo, de manera dirigida fundamentalmente a los profesionales sanitarios que trabajan en la atención primaria.

Elaborado por el Ministerio de Sanidad y Consumo y las Comunidades Autónomas, en el año 2003 se aprobó el Plan Nacional de Prevención y Control del Tabaquismo ${ }^{50}$, que permite establecer un marco común de actuación frente a uno de los principales problemas de salud pública, el tabaquismo, que representa la primera causa de enfermedad y muerte prevenible en España, y es un factor de riesgo cardiovascular de primer orden. Este Plan pretende recoger aquellos aspectos fundamentales para el abordaje global del tabaquismo, con unos objetivos consensuados y alcanzables desde distintas estrategias.

Distintas Sociedades Científicas españolas han publicado valiosos documentos de recomendaciones para la prevención de las enfermedades cardiovasculares y para el control de sus principales factores de riesgo. Hay que destacar los documentos de la Sociedad Española de Arteriosclerosis, que en 1989 publicó las Recomendaciones para la prevención de la arteriosclerosis en Espa$\tilde{n} a^{51}$. En 1994, en colaboración con la Sociedad Española de Medicina Interna y la Liga para la Lucha contra la Hipertensión Arterial, se publicaron las Recomendaciones para la prevención primaria de la enfermedad cardiovascular ${ }^{52}$; y, junto con la Sociedad Española de Cardiología, las Recomendaciones para el tratamiento de la hiperlipidemia en pacientes con cardiopatía isquémi$\mathrm{ca}^{53}$. La Sociedad Española de Diabetes y la Sociedad Española de Arteriosclerosis publicaron el documento de consenso Dislipemia diabética en $1998^{54}$. Por su parte, el grupo de trabajo de Dislipemias de la Sociedad Española de Medicina de Familia y Comunitaria publicó su informe sobre el manejo de las dislipemias en atención primaria en $1997^{55}$.

Centradas en la hipertensión, se pueden mencionar la Guía sobre el diagnóstico y el 
tratamiento de la hipertensión arterial en España, publicada en 2002 por la Sociedad Española de Hipertensión-Liga Española para la Lucha contra la Hipertensión Arterial $^{56}$, y las Guías de práctica clínica en hipertensión arterial de la Sociedad Española de Cardiología, publicadas en $2000^{57}$ y actualizadas en $2003^{58}$.

Sobre el aspecto clave en la salud cardiovascular de la dieta, el sobrepeso y la obesidad, también se dispone de distintos documentos de recomendaciones. En 1994 la Sociedad Española de Arteriosclerosis publicó sus Recomendaciones sobre dieta y enfermedades cardiovasculares ${ }^{59}$. Por su parte, la Sociedad Española de Nutrición Comunitaria ha editado sus completas segundas Guías alimentarias para la población española en el año $2001^{60}$. La Sociedad Española para el Estudio de la Obesidad publicó en 2000 su Consenso para la evaluación del sobrepeso y la obesidad y el establecimiento de criterios de intervención terapéutica $^{61}$.

Como magnífico ejemplo de actuaciones preventivas estructuradas desarrolladas en todas las comunidades Autónomas españolas desde 1988 se desarrolla el Programa de Actividades Preventivas y de Promoción de la Salud en Atención Primaria (PAPPS), de la Sociedad Española de Medicina Familiar y Comunitaria. El PAPPS orienta sus actividades hacia la prevención de las principales enfermedades crónicas, entre ellas las enfermedades cardiovasculares, a través de la lucha contra sus principales factores de riesgo, mediante recomendaciones y criterios consensuados de actuación ${ }^{62}$.

En el ámbito de los niños y jóvenes en 1991se publicó el Informe sobre el colesterol en niños y adolescentes españoles, elaborado por las Sociedades Españolas de Arteriosclerosis, Cardiología, Pediatría, Nutrición y Medicina Preventiva ${ }^{63}$.

Finalmente, se puede reseñar la publicación del Plan Integral de Cardiopatía Isqué- mica 2004-200764, elaborado de manera conjunta entre el Estado y las Comunidades Autónomas, para el establecimiento de estándares y modelos de atención sobre prevención, diagnóstico, tratamiento y rehabilitación.

\section{ADAPTACIÓN ESPAÑOLA DE LA GUÍA EUROPEA DE PREVENCIÓN CARDIOVASCULAR}

En este número de la Revista Española de Salud Pública, junto con la traducción de la Guía Europea $^{34}$, se publica la Adaptación española de la Guía Europea de Prevención Cardiovascular preparada por el Comité Español Interdisciplinario para la Prevención Cardiovascular ${ }^{65}$, compuesto por once sociedades científicas españolas involucradas en la prevención cardiovascular, y la administración sanitaria. De esta forma se da cumplimiento a la pretensión de la Guía Europea de impulsar el desarrollo de guías nacionales para la prevención cardiovascular. Este documento adapta la guía de prevención cardiovascular elaborada por las sociedades europeas a las características epidemiológicas de las enfermedades cardiovasculares en España y a nuestro sistema sanitario. Por ello, propone utilizar la versión para países de baja incidencia del modelo SCORE para la valoración del riesgo cardiovascular, e introduce algunas modificaciones:

- Respecto a la extrapolación del riesgo a los 60 años de edad, propuesta por la Guías Europeas, el CEIPC recomienda que en aquellas personas jóvenes con varios factores de riesgo pero no consideradas de alto riesgo a causa de su edad, antes que hacer la extrapolación, se debería hacer énfasis en el consejo y los cambios en los estilos de vida.

- En personas asintomáticas, la Adaptación española señala que, en nuestro entorno, el colesterol total debería ser 
menor de $200 \mathrm{mg} / \mathrm{dl}(5,2 \mathrm{mmol} / \mathrm{l})$ y el cLDL menor de $130 \mathrm{mg} / \mathrm{dl}(3,4$ $\mathrm{mmol} / \mathrm{l})$. A diferencia de lo propuesto por las Guías Europeas, que dicen que en general la colesterolemia debe ser menor de $190 \mathrm{mg} / \mathrm{dl}(5 \mathrm{mmol} / \mathrm{l})$ y el cLDL menor de $115 \mathrm{mg} / \mathrm{dl}$ (3 mmol/l).

- En personas asintomáticas con riesgo alto (riesgo de muerte cardiovascular igual o mayor del 5\% a los 10 años) recomienda en nuestro entorno dieta y tratamiento hipolipemiante cuando los niveles de colesterol total y cLDL sean iguales o mayores de $200 \mathrm{mg} / \mathrm{dl}$ y de $130 \mathrm{mg} / \mathrm{dl}$ respectivamente. Mientras que las Guías Europeas lo indican cuando los niveles de colesterol total y colesterol LDL sean iguales o mayores de 190 mg/dl y de 115 mg/dl respectivamente.

- Se prioriza que todos los fumadores reciban consejo firme profesional para dejar de fumar, señalando que éste constituye el factor más importante para poner en marcha el proceso de abandono del tabaco en personas con enfermedades cardiovasculares.

- Incluye un anexo con recomendaciones dietéticas adaptadas a nuestro entorno y con criterios de derivación o consulta con el especialista de los pacientes hipertensos y dislipémicos.

\section{CONSIDERACIONES FINALES}

Aunque todavía no se puede explicar una parte de la morbilidad y mortalidad cardiovascular, por lo que se debe profundizar en la investigación de otros factores de riesgo, sí se conoce lo suficiente sobre los principales factores de riesgo cardiovascular como para poder actuar eficientemente sobre ellos.

Dado que la prevalencia de los factores de riesgo cardiovascular es elevada, la demanda asistencial que generan es alta en un Sistema Nacional de Salud universal y gratuito como es el español, en el que los recursos disponibles son limitados y los resultados de las intervenciones realizadas por el personal sanitario son discretos cuando se valora su efecto sobre el total de la población y en personas de bajo riesgo, haciéndose necesario establecer un orden de prioridades en las actuaciones preventivas cardiovasculares.

La forma más razonable y coste-efectiva de determinar las prioridades de prevención en las personas asintomáticas es mediante el riesgo cardiovascular, ya que permite establecer una asignación de los recursos en función de las necesidades, entendiendo como tales el riesgo de sufrir una enfermedad cardiovascular.

Para estimar el riesgo cardiovascular se pueden emplear distintas tablas. La utilidad práctica de las tablas de riesgo es seleccionar a los grupos de personas tributarias de estrategias de prevención primaria cardiovascular acordes con su riesgo promedio absoluto, más que estimar su riesgo cardiovascular individual, ya que en la actualidad no se es capaz de diagnosticar por anticipado la aparición de un episodio cardiovascular agudo en una persona determinada. Sólo se puede avanzar su probabilidad de enfermar o morir por causa cardiovascular. Las tablas basadas en el estudio de seguimiento de Framingham, aunque puedan sobreestimar el riesgo absoluto cuando se utilicen en poblaciones con baja morbimortalidad, parece claro que ordenan de forma adecuada a las personas en cuanto a su riesgo coronario, y por tanto, cumplen bien con su finalidad.

El debate sobre qué tabla de riesgo utilizar es bueno en la medida que refuerza la necesidad de emplear el riesgo cardiovascular y favorece su conceptualización y, en definitiva, puede ayudar a su uso. Sin embargo, puede originar cierto grado de confusión y desorientación sobre la cantidad de tablas exis- 
tentes, unas que aparecen y desaparecen, otras propuestas por un único grupo de trabajo, otras circunscritas a un ámbito geográfico determinado, otras restringidas en su uso a un sólo factor de riesgo, como puede ser la hipertensión arterial. Todo ello puede llevar a la tentación de utilizar la última tabla que aparece, produciendo un zigzagueante vaivén que produce vértigo y, en definitiva, teniendo que cambiar de tabla cada pocos meses sin suficiente razón que lo justifique, dificultando así la generalización del uso del riesgo cardiovascular en la valoración clínica cotidiana del paciente. Otro posible efecto desfavorable de la proliferación de tablas puede ser el contrario, el desánimo y la actitud de no usar ninguna hasta que se clarifique el tema. No hay que olvidar que las tablas no son más que una herramienta para ayudar en la toma de decisiones sobre el tratamiento y seguimiento en prevención primaria. Las tablas no son un fin en sí mismo, sólo son un medio para ayudar a llevar a cabo la prevención cardiovascular. El objetivo es mejorar la salud cardiovascular de la población, para ello se realizarán actividades preventivas que estarán guiadas por unas recomendaciones que utilizarán el riesgo cardiovascular, siendo la tabla una mera herramienta para la valoración de este riesgo cardiovascular en las personas asintomáticas. Como se ha expuesto con anterioridad, se dispone de numerosas y valiosas recomendaciones, y la adaptación española de la Guía Europea de Prevención Cardiovascular (65), ampliamente consensuada por las sociedades científicas españolas, es un magnifico ejemplo. Además del rigor metodológico en la elaboración de las recomendaciones y su adecuación a la situación del ámbito donde se van a desarrollar, es fundamental su difusión para que sean conocidas y, sobre todo, para que sean capaces de influir en la práctica clínica cotidiana de quienes las conocen. Por tanto, ha llegado el momento de ponerla en práctica, centrando los esfuerzos en este propósito y dejando el tiempo suficiente como para que se generalice su uso.

\section{BIBLIOGRAFÍA}

1. Organización Mundial de la Salud. Informe sobre la Salud en el Mundo 2003: Forjemos el futuro. Ginebra: OMS; 2003.

2. Sans S, Kesteloot H, Kromhout D, et al. The burden of cardiovascular disease mortality in Europe. Task Force on the European Society of Cardiology on Cardiovascular Mortality and Morbidity Statistics in Europe. Eur Heart J 1997; 18:1231-48.

3. Tunstall-Pedoe H, Kuulasmaa K, Mahonen M, Tolonen H, Ruokokoski E, Amouyel P et al. Contribution of trends in survival and coronary-event rates to changes in coronary heart disease mortality: 10-year results from 37 WHO MONICA Project populations. Lancet 1999; 353: 1547-57.

4. Kuulasmaa K, Tunstall-Pedoe H, Dobson A, Fortmann S, Sans S, Tolonen H, Evans A, Tuohmilehto $\mathrm{J}$, for the WHO MONICA Project. Estimating the contribution of changes in classical risk factors to trends in coronary-event rates across the WHO MONICA Project populations. Lancet 2000; 355: 675-87.

5. Villar Álvarez F, Banegas JR, Donado J, Rodríguez Artalejo F. Las enfermedades cardiovasculares y sus factores de riesgo en España: hechos y cifras. Madrid: Sociedad Española de Arteriosclerosis; 2003.

6. Villar Álvarez F, Banegas JR, Rodríguez Artalejo F, Rey Calero J. Mortalidad cardiovascular en España y sus Comunidades Autónomas (19751992). Med Clin (Barc) 1998; 110 (09): 321-7.

7. Banegas JR, Díez Gañán L, Rodríguez Artalejo F, González Enríquez J, Graciani Pérez-Regadera A, Villar Álvarez F. Mortalidad atribuible al tabaquismo en España en 1998. Med Clin (Barc) 2001; 117(18): 692-4.

8. García J, Elosua R, Tormo Díaz MJ, Audicana Uriarte C, Zurriaga O, Segura A, et al. Letalidad poblacional por infarto agudo de miocardio. Estudio IBERICA. Med Clin (Barc) 2003;121(16):606-12.

9. Organización Mundial de la Salud. Los objetivos de la Salud para Todos. Estrategia Regional Europea. Madrid: Ministerio de Sanidad y Consumo; 1986.

10. Organización Mundial de la Salud. Salud 21. Salud para Todos en el siglo XXI. Madrid: Ministerio de Sanidad y Consumo; 1999.

11. Junta Asesora de la Conferencia Internacional de Salud Cardiovascular. La Declaración de Victoria. 
Cerrando la brecha: Ciencia y Política en Acción. Rev Sanid Hig Pública 1993; 67(02): 77-116.

12. Organización Mundial de la Salud. Prevención de la cardiopatía coronaria. Informe Técnico nº 678 . Ginebra: OMS, 1982.

13. Organización Mundial de la Salud. Prevención y lucha contra las enfermedades cardiovasculares en la comunidad. Informe Técnico $\mathrm{n}^{\circ} 732$. Ginebra: OMS; 1986.

14. Organización Mundial de la Salud. Dieta, nutrición y prevención de enfermedades crónicas. Informe Técnico nº 797. Ginebra: OMS; 1990.

15. Organización Mundial de la Salud. Dieta, nutrición y prevención de enfermedades crónicas. Informe Técnico nº 916. Ginebra: OMS; 2003.

16. Organización Mundial de la Salud. Hipertensión y Cardiopatía Coronaria. Informe Técnico no 168. Ginebra: OMS; 1959.

17. Organización Mundial de la Salud. Hipertensión Arterial y Cardiopatía Isquémica. Informe Técnico n 231. Ginebra: OMS; 1962.

18. Organización Mundial de la Salud. Hipertensión Arterial. Informe Técnico no 628. Ginebra: OMS; 1978.

19. Organización Mundial de la Salud. Control de la Hipertensión. Informe Técnico $n^{\circ}$ 862. Ginebra: OMS; 1996.

20. World Health Organisation-International Society of Hypertension. 1999 World Health OrganisationInternational Society of Hypertension Guidelines for the Management of Hypertension. Guidelines Subcommittee. J Hypertension 1999; 17:151-83.

21. World Health Organization, International Society of Hypertension Writing Group. 2003 World Health Organization (WHO)/International Society of Hypertension (ISH) statement on management of hypertension. J Hypertens 2003; 21(11): 198392.

22. National Cholesterol Education Program. Report of the National Cholesterol Education Program Expert Panel on Detection, Evaluation, and Treatment of High Blood Cholesterol in Adults: the Expert Panel. Arch Intern Med 1988; 148:36-69.

23. National Cholesterol Education Program. Report of the Expert Panel on Blood Cholesterol Levels in Children and Adolescents. Bethesda, MD: U.S. Public Health Service, National Institutes of Health; 1991.
24. National Cholesterol Education Program. Second Report of the Expert Panel on Detection, Evaluation and Treatment of High Blood Cholesterol in Adults (Adult Treatment Panel II). Circulation 1994; 89:1329-445.

25. National Cholesterol Education Program. Executive Summary of The Third Report of The National Cholesterol Education Program (NCEP) Expert Panel on Detection, Evaluation, And Treatment of High Blood Cholesterol In Adults (Adult Treatment Panel III).JAMA 2001;285(19):2486-97.

26. Joint National Committee on Detection, Evaluation, and Treatment of High Blood Pressure. The Sixth Report of the Joint National Committee on Prevention, Detection, Evaluation, and Treatment of High Blood Pressure (JNC VI). Arch Intern Med 1997; 157:2413-46.

27. Chobanian AV, Bakris GL, Black HR, Cushman WC, Green LA, Izzo JL, et al. The Seventh Report of the Joint National Committee on Prevention, Detection, Evaluation, and Treatment of High Blood Pressure. The JNC 7 Report. JAMA 2003; 289(19):2560-72.

28. Guidelines Committee. 2003 European Society of Hypertension-European Society of Cardiology guidelines for the management of arterial hypertension. J Hypertens 2003; 21(06): 1011-53.

29. Williams CL, Hayman LL, Daniels SR, Robinson TN, Steinberger J, Paridon S, et al. Cardiovascular Health in Childhood. A Statement for Health Professionals From the Committee on Atherosclerosis, Hypertension, and Obesity in the Young (AHOY) of the Council on Cardiovascular Disease in the Young, American Heart Association. Circulation 2002; 106(01): 143-60.

30. Pearson TA, Blair SN, Daniels SR, Eckel RH, Fair JM, Fortmann SP, et al. AHA Guidelines for Primary Prevention of Cardiovascular. Disease and Stroke: 2002 Update. Consensus Panel Guide to Comprehensive Risk Reduction for Adult Patients Without Coronary or Other Atherosclerotic Vascular Diseases. Circulation 2002; 106(03): 388-91.

31. Eyre H, Kahn R, Robertson RM, ACS/ADA/AHA Collaborative Writing Committee. Preventing Cancer, Cardiovascular Disease, and Diabetes. A Common Agenda for the American Cancer Society, the American Diabetes Association, and the American Heart Association. Circulation 2004; 109(25): 3244-55.

32. Study Group of the European Atherosclerosis Society. Strategies for the prevention of coronary heart disease. Eur Heart J 1987; 8:77-88. 
33. Prevention of coronary heart disease: Scientific background and new clinical guidelines. Recommendations of the European Atherosclerosis Society prepared by the International Task Force for Prevention of Coronary Heart Disease. Nut Metab Cardiovasc Dis 1992;2:113-156.

34. De Backer G, Ambrosioni E, Borch-Johnsen K, Brotons C, Cifkova R, Dallongeville J, et al. Guía Europea de prevención cardiovascular en la práctica clínica. Tercer Grupo de Trabajo de las Sociedades europeas y otras sociedades sobre prevención cardiovascular en la práctica clínica. Rev Esp Salud Pública 2004;78(04):439-456.

35. Pyörälä K, De Backer G, Graham I, Poole-Wilson P, Wood D, on behalf of the Task Force. Prevention of coronary heart disease in clinical practice. Recommendations of the Tásk Force of the European Society of Cardiology, European Atheroselerosis Society and European Society of Hypertension. Eur Heart J 1994; 15: (301-3); Atherosclerosis 1994; 110:121-61.

36. Wood D, De Backer G, Faegerman O, Graham I, Mancia G, Pyörala K. Prevention of coronary heart disease in clinical practice. Recommendations of the Second Joint Task Force of European and other Societies on Coronary Prevention. Eur Heart J 1998; 19:1434-503.

37. De Backer G, Ambrosioni E, Borch-Johnsen K, Brotons C, Cifkova R, Dallongeville J, et al. European guidelines on cardiovascular disease prevention in clinical practice. Third Joint Task Force of European and other Societies on Cardiovascular Disease Prevention in Clinical Practice. Eur Heart J 2003; 24(17): 1601-10.

38. Conroy RM, Pyörälä K, Fitzgerald AP, Sans S, Menotti A, De Backer G, et al. Estimation of ten-year risk of fatal cardiovascular disease in Europe: the SCORE project. Eur Heart J 2003; 24:987-1003.

39. Wood DA, Durrington P, McInnes G, Poulter N, Rees A, Wray R. Joint British recommendations on prevention of coronary heart disease in clinical practice. Heart 1998;80(suppl):S1-29.

40. British Hypertension Society. Guidelines for hypertension management 1999: summary. BMJ 1999; 319:630-5.

41. Scottish Intercollegiate Guidelines Network (SIGN). Lipids and the Primary Prevention of Coronary Heart Disease. Edimburgo: SIGN; 1999.

42. Scottish Intercollegiate Guidelines Network (SIGN). Secondary Prevention of Coronary Heart
Disease following Myocardial Infarction. Edimburgo: SIGN; 2000.

43. Ministerio de Sanidad y Consumo, Sociedad Española de Cardiología. Consenso para el control de la Colesterolemia en España. Rev Sanid Hig Pública 1989; 63:109-9.

44. Ministerio de Sanidad y Consumo, Sociedad Española de Cardiología, Sociedad Española de Arteriosclerosis. Control de la colesterolemia en España, 2000. Un instrumento para la prevención cardiovascular. Rev Esp Salud Pública 2000;74(3): 215-53.

45. Ministerio de Sanidad y Consumo, Liga Española para la Lucha Contra la Hipertensión Arterial. Consenso para el control de la Hipertensión Arterial en España. Rev Sanid Hig Pública 1990; 64:439-77.

46. Ministerio de Sanidad y Consumo, Sociedad-Liga Española para la Lucha Contra la Hipertensión Arterial. Control de la hipertensión arterial en España, 1996. Rev Esp Salud Pública 1996;70(02): 139-210.

47. Banegas JR, Villar F. Políticas de promoción de la salud. Rev Sanid Hig Pública 1993; 67(02): 125-8.

48. Brotons C, Server M, Pintó X, Roura P, MartínZurro A. Impacto de los consensos para el control de la colesterolemia y la hipertensión en España. Med Clin (Barc) 1997; 108 (01): 9-15.

49. Grupo de Trabajo de Prevención Primaria Cardiovascular. Directrices para la elaboración de programas de prevención primaria de enfermedades cardiovasculares. Rev Sanid Hig Pública 1993; 67(01): 5-22.

50. Ministerio de Sanidad y Consumo. Plan Nacional de Prevención y Control del Tabaquismo. Rev Esp SaludPública2003;77(04):441-71.

51. Carmena R, Ros E, Gómez Gerique JA, Masana L, Ascaso JF. Recomendaciones para la prevención de la arteriosclerosis en España. Documento Oficial de la Sociedad Española de Arteriosclerosis. Clin Invest Arterioscl 1989; 1:1-9.

52. Sociedad Española de Arteriosclerosis, Sociedad Española de Medicina Interna, Liga para la Lucha contra la Hipertensión Arterial. Recomendaciones para la prevención primaria de la enfermedad cardiovascular. Clin Invest Arterioscl 1994; 6(02): 62102.

53. Sociedad Española de Arteriosclerosis, Sociedad Española de Cardiología. Evidencias clinicoexperimentales y recomendaciones para el tratamiento 
de la hiperlipidemia en pacientes con cardiopatía isquémica. Clin Invest Arterioscl 1994; 6(02): 10311.

54. González Santos P, Herrera Pombo JL, Ascaso JF, Escobar Jiménez F, Gómez Gerique JA, Jiménez Perepérez JA, et al. Dislipemia diabética: Documento de consenso de la Sociedad Española de Diabetes y la Sociedad Española de Arteriosclerosis. Av Diabetol 1998;14:33-43.

55. Lago Deibe F, Abad Vivas Pérez JJ, Álvarez Cosmea A, Blasco Valle M, Álamo Alonso AJ, Llor Vila C, et al. Dislipemias. Manejo de las dislipemias en atención primaria. Madrid: SEMFYC; 1997.

56. Sociedad Española de Hipertensión-Liga Española para la Lucha contra la Hipertensión Arterial (SEHLELHA). Guía sobre el diagnóstico y el tratamiento de la hipertensión arterial en España 2002. Hipertensión 2002;19 (Supl 3):1-74.

57. Lombera Romero F, Barrios Alonso V, Soria Arcos F, Placer Peralta L, Cruz Fernández JM, Tomás Abadal L, et al. Guías de práctica clínica de la Sociedad Española de Cardiología en hipertensión arterial. Rev Esp Cardiol 2000; 53(05): 66-90.

58. González-Juanatey JR, Mazón Ramos P, Soria Arcos F, Barrios Alonso V, Rodríguez Padial L, Bertomeu Martínez V. Actualización (2003) de las Guías de práctica clínica de la Sociedad Española de Cardiología en hipertensión arterial. Rev Esp Cardiol 2003; 56(05): 487-97.
59. Mata P, de Oya M, Pérez-Jiménez F, Ros Rahola E. Dieta y enfermedades cardiovasculares. Recomendaciones de la Sociedad Española de Arteriosclerosis. Clin Invest Arterioscl 1994; 6(02): 43-61.

60. Sociedad Española de Nutrición Comunitaria Guías alimentarias para la población española. Madrid: SENC, 2001.

61. Sociedad Española para el Estudio de la Obesidad (SEEDO). Consenso SEEDO'2000 para la evaluación del sobrepeso y la obesidad y el establecimiento de criterios de intervención terapéutica. Med Clin (Barc) 2000; 115(15): 587-97.

62. Sociedad Española de Medicina Familiar y Comunitaria. Programa de Actividades Preventivas y de Promoción de la Salud en Atención Primaria. Actualización 2003. Aten Primaria 2003;32(Supl 2):1-158.

63. Plaza I. Grupo de Expertos de las Sociedades Españolas de Arteriosclerosis, Cardiología, Pediatría, Nutrición y Medicina Preventiva. Informe sobre el colesterol en niños y adolescentes españoles. Clin Invest Arteriosclerosis 1991; 3:67-75.

64. Ministerio de Sanidad y Consumo. Plan Integral de Cardiopatía Isquémica 2004-2007. Madrid: Ministerio de Sanidad y Consumo; 2003.

65. Comité Español Interdisciplinario para la Prevención Cardiovascular. Adaptación española de la Guía Europea de Prevención Cardiovascular. Rev Esp Salud Publica 2004;78(04):435-438. 